\title{
Deformidade dos ossos pélvicos e sua correlação com o ângulo centro-borda acetabular*
}

\section{Pelvic Bone Deformity and Its Correlation with Acetabular Center-edge Angle}

\author{
Bruno Dutra Roos ${ }^{1}$ Milton Valdomiro Roos ${ }^{1}$ Antero Camisa Júnior ${ }^{1}$ \\ Ezequiel Moreno Ungaretti Lima ${ }^{1}$ Darby Lira Tisatto ${ }^{1}$ Marcel Cruz Argoud ${ }^{1}$ \\ ${ }^{1}$ Grupo de Quadril do Hospital Ortopédico de Passo Fundo, Faculdade \\ de Medicina da Universidade de Passo Fundo, Passo Fundo, RS, Brasil \\ Rev Bras Ortop 2020;55(2):239-246.
Endereço para correspondência Bruno Dutra Roos, MD, Grupo de Quadril do Hospital Ortopédico de Passo Fundo, Faculdade de Medicina da Universidade de Passo Fundo, Av. Sete de Setembro, 817, Centro, Passo Fundo, RS 99010-121, Brasil (e-mail: brunodroos@gmail.com).

\section{Resumo}

Palavras-chave

- luxação do quadril

- acetábulo

- cabeça do fêmur
Objetivos O objetivo do presente trabalho é avaliar a deformidade dos ossos pélvicos e sua correlação com ângulo centro-borda acetabular (CE).

Métodos Foi realizado um estudo prospectivo caso-controle, entre agosto de 2014 e abril de 2015. Os critérios de inclusão foram pacientes consecutivos com idades entre 20 e 60 anos. Os critérios de exclusão foram: doença metabólica, cirurgia prévia de quadril ou coluna, radiografia evidenciando artrose de quadril $\geq$ Tönnis 2 , displasia do desenvolvimento do quadril (DDQ) severa, sobrecobertura acetabular global, sinal do cruzamento das linhas acetabulares, deformidades decorrentes de epifisiólise ou Legg-Perthes-Calveé, e radiografia sem qualidade adequada. Foram avaliados na radiografia anteroposterior (AP) de pelve: o ângulo CE, índice acetabular (IA), sinal do cruzamento das linhas acetabulares, mensuração do eixo horizontal e vertical da hemipelve superior e inferior $(\mathrm{H} 1$ : Linha Horizontal 1, hemipelve superior; H2: Linha Horizontal 2, hemipelve superior; V1: Linha Vertical, hemipelve superior; HR: Linha Horizontal, hemipelve inferior; VR: Linha Vertical, hemipelve inferior). As mensurações $\mathrm{H} 1, \mathrm{H} 2, \mathrm{~V} 1, \mathrm{HR}$ e VR foram consideradas assimétricas quando, na comparação de uma hemipelve em relação ao lado contralateral, evidenciou-se uma diferença $>5 \mathrm{~mm}$. Os pacientes foram separados em dois grupos: controle e grupo 1 . Resultados O total de pacientes avaliados no período foi de 228 (456 quadris). De acordo com os critérios estabelecidos, foram incluídos neste estudo 93 pacientes. A idade média foi de 39,9 anos (20 a 60 anos, desvio padrão [DP] $=10,5$ ), e o ângulo $C E$ médio do quadril direito foi de $31,5^{\circ}\left(20^{\circ}\right.$ a $\left.40^{\circ}\right)$ e do esquerdo de $32,3^{\circ}\left(20^{\circ}\right.$ a $\left.40^{\circ}\right)$. Um total de 38 pacientes foi incluído no grupo controle, sendo que com relação à $\mathrm{H} 1$, foi constatada aferição assimétrica em 4 casos (10,5\%), H2 em 5 (13,1\%), V1 em 7 (18,4\%), HR em 5 (13,1\%), e VR em 1 caso (2,63\%). No grupo 1, foram incluídos 55 pacientes, sendo que com relação à $\mathrm{H} 1$, foi constatada aferição assimétrica em 24 casos (43,6\%), H2 em 50 (90,9\%), V1 em 28 (50,9\%), HR em 16 (29,09\%), e VR em 8 casos (14,5\%). Na
Trabalho feito no Grupo de Quadril do Hospital Ortopédico de Passo Fundo, Faculdade de Medicina da Universidade de Passo Fundo, Passo Fundo, RS, Brasil. recebido

30 de Novembro de 2018

aceito

22 de Janeiro de 2019
DOI https://doi.org/

10.1055/s-0039-3400516. ISSN 0102-3616.
Copyright $\odot 2020$ by Sociedade Brasileira License terms de Ortopedia e Traumatologia. Published by Thieme Revinter Publicações Ltda, Rio de Janeiro, Brazil 


\begin{abstract}
Keywords

- hip dislocation

- acetabulum

- femur head

Objective The purpose of the present study was to evaluate the pelvic bone deformities and its correlation with the acetabular center-edge (CE) angle.

Methods Between August 2014 and April 2015, we prospectively evaluated patients aged between 20 and 60 years old. The exclusion criteria were: metabolic disease, previous hip or spine surgery, radiograph showing hip arthrosis $\geq$ Tönnis two, severe hip dysplasia, global acetabular overcoverage, acetabular crossover sign, hip deformities from slipped capital femoral epiphysis (SCFE) or Leg-Perthes-Calveé, and bad quality radiographs. At anteroposterior (AP) pelvic radiographs, we have evaluated: the CE angle, the acetabular index (IA), the acetabular crossover sign, the vertical and horizontal superior and inferior pelvic axis ( $\mathrm{H} 1$ : Horizontal line 1, superior pelvic axis; H2: Horizontal line 2, superior pelvic axis; V1: Vertical line, superior pelvic axis; HR: Horizontal line, inferior pelvic axis; VR: Vertical line, inferior pelvic axis). The superior and inferior pelvic axis were considered asymmetric when there was a difference $\geq$ $5 \mathrm{~mm}$ between both sides. Patients were divided into two groups: control and group 1. Results A total of 228 patients (456 hips) were evaluated in the period. According to the established criteria, 93 patients were included. The mean age was 39.9 years old (20 to 60 years old, standard deviation $[S D]=10,5)$, and the mean $C E$ angle in the right hip was $31.5^{\circ}\left(20^{\circ}\right.$ to $\left.40^{\circ}\right)$, and in the left $32.3^{\circ}\left(20^{\circ}\right.$ to $\left.40^{\circ}\right)$. The control group had 38 patients, with asymmetric $\mathrm{H} 1$ in 4 cases (10.5\%), $\mathrm{H} 2$ in 5 (13.1\%), $\mathrm{V} 1$ in 7 (18.4\%), HR in $5(13.1 \%)$ and VR in 1 (2.63\%). Group 1 had 55 patients, with asymmetric $\mathrm{H} 1$ in 24 cases (43.6\%), H2 in 50 (90.9\%), V1 in 28 (50.9\%), HR in 16 (29.09\%) and VR in 8 (14.5\%). Comparing both groups, there was statistical significance for $\mathrm{H} 1, \mathrm{H} 2$ and $\mathrm{V} 1$ asymmetry $(p<0.001)$.

Conclusion In the present paper, we observed the correlation between variation in the acetabular CE angle and asymmetry of the superior hemipelvis. The present authors believe that a better understanding of the pelvic morphologic alterations allows a greater facility in the diagnosis of hip articular deformities.
\end{abstract}

comparação entre o grupo controle e o grupo 1, observou-se diferença estatisticamente significativa para a assimetria das mensurações H1, H2 e V1 $(p<0,001)$.

Conclusão No presente trabalho, evidenciou-se correlação entre variação do ângulo CE acetabular e assimetria da hemipelve superior. Os presentes autores acreditam que o melhor entendimento das alterações morfológicas pélvicas permite uma maior facilidade no diagnóstico das deformidades articulares do quadril.

\section{Introdução}

Existe evidência crescente na literatura da associação de alterações na morfologia óssea da região do quadril e o desenvolvimento de sintomas, além da possibilidade de evolução para degeneração condral articular. Estas alterações podem estar relacionadas ao fêmur, ao acetábulo ou a ambos. ${ }^{1}$ No lado acetabular, como alterações morfológicas frequentes pode-se encontrar a sobrecobertura (impacto femoroacetabular [IFA] tipo Pincer) e a deficiência de cobertura (displasia do desenvolvimento do quadril [DDQ]). 2,3

A sobrecobertura acetabular pode ser global ou focal. Sobrecobertura global é definida na radiografia anteroposterior (AP) de pelve por ângulo centro-borda $(\mathrm{CE})^{4}>40^{\circ}$ associado a excesso de cobertura da cabeça femoral pela parede anterior e posterior do acetábulo. ${ }^{5}$ Sobrecobertura focal é definida pela presença de retroversão acetabular, que é uma alteração morfológica na qual há desvio estrutural do acetábulo no plano sagital em direção posterolateral. Radiograficamente, a retroversão acetabular é representada pela presença do sinal do cruzamento das linhas acetabulares. $3,6,7$ Ambas as alterações têm sido associadas ao impacto dinâmico entre o rebordo acetabular e a transição cabeça-colo femoral, podendo resultar em lesões acetabulares da cartilagem posteroinferior e do lábio, além de dor. ${ }^{8}$

Na deficiência de cobertura acetabular, uma área de contato reduzida entre a cabeça femoral e o acetábulo gera força de cisalhamento excessiva na junção condrolabral acetabular, podendo levar ao surgimento de sintomas e degeneração condral em longo prazo. Esta deficiência mais comumente é anterossuperior no acetábulo, e o diagnóstico de DDQ é realizado quando o ângulo $\mathrm{CE}^{4}$ for $<25^{\circ}$ em radiografia $\mathrm{AP}$ de pelve., ${ }^{4,9}$

Alguns autores têm proposto que os distúrbios do desenvolvimento do quadril não afetam somente o fêmur proximal e o acetábulo, como é evidenciado em patologias como IFA e DDQ mas sim em toda a pelve. ${ }^{1,10}$ Porém, não há consenso 
sobre quais deformidades ósseas pélvicas correlacionam-se com as alterações morfológicas acetabulares. Os presentes autores acreditam que as alterações estruturais ósseas da pelve e anormalidades acetabulares não são achados isolados, e fazem parte de um continuum de alterações estruturais do desenvolvimento da pelve.

O objetivo do presente trabalho é avaliar a deformidade dos ossos pélvicos e sua correlação com o ângulo CE.

\section{Materiais e Métodos}

Foi realizado um estudo prospectivo caso-controle entre agosto de 2014 e abril de 2015. O trabalho obteve aprovação do Comitê de Ética em Pesquisa, e todos os participantes assinaram o Termo de Consentimento.

Os participantes foram convidados ao estudo voluntariamente. Os critérios de inclusão foram pacientes consecutivos com idade entre 20 e 60 anos. Os critérios de exclusão foram: doença metabólica, cirurgia prévia de quadril ou coluna, radiografia evidenciando artrose de quadril $\geq$ Tönnis 2 , DDQ severa (ângulo $\mathrm{CE}<20^{\circ}$ ), ${ }^{4,9}$ sobrecobertura acetabular global (ângulo CE $>40^{\circ}$ e/ou índice acetabular $<0^{\circ}$ ), ${ }^{5}$ sinal do cruzamento das linhas acetabulares (sugerindo retroversão acetabular), assimetria de ângulo $\mathrm{CE}<5^{\circ}$, deformidades decorrentes de epifisiólise ou Legg-Perthes-Calveé, e radiografia sem qualidade adequada. ${ }^{11}$

Os pacientes selecionados realizaram radiografia de pelve na incidência $\mathrm{AP}$ com os pés em $15^{\circ}$ de rotação interna, com o tubo a $120 \mathrm{~cm}$ de distância do filme e com o raio direcionado ao ponto central entre a borda superior da sínfise púbica e uma linha horizontal conectando ambas as espinhas ilíacas anterossuperiores. A distância entre o cóccix e a sínfise púbica, além do seu alinhamento, foram fatores considerados para avaliar a qualidade das radiografias. ${ }^{11}$

Foram avaliados no estudo radiográfico em ambas as hemipelves: o ângulo $\mathrm{CE}$, índice acetabular (IA), sinal do cruzamento das linhas acetabulares, mensuração do eixo horizontal e vertical da hemipelve superior (asas do ilíaco H1, H2 e V1), mensuração do eixo horizontal e vertical da hemipelve inferior (ramos ísquio e ílio púbicos - HR e VR). A definição do método para aferição dos eixos pélvicos está exemplificada na -Figura $\mathbf{1}$.
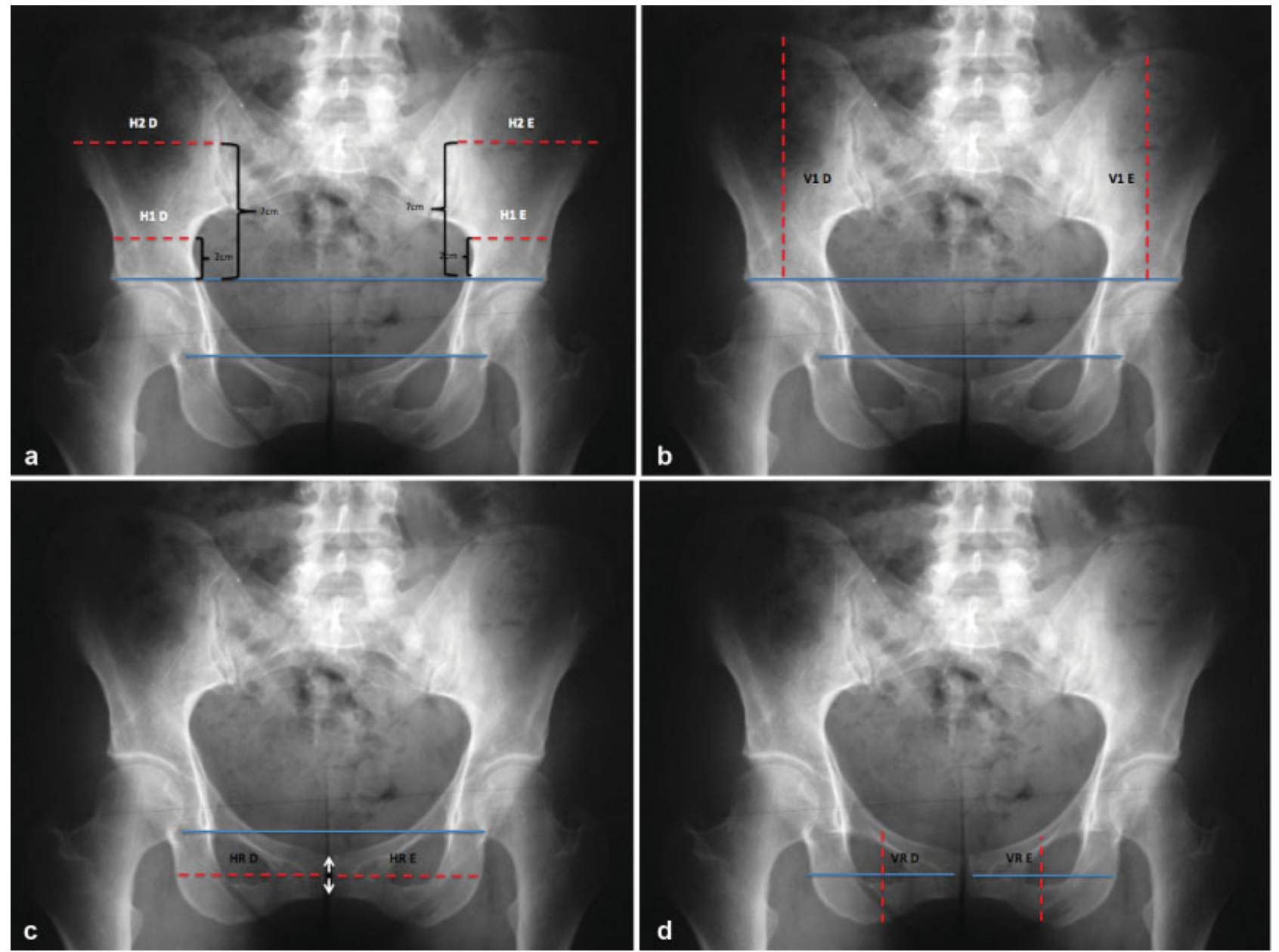

Fig. 1 Exemplificação do método de aferição dos eixos pélvicos bilateralmente. Todas as linhas (H1, H2, V1, HR e VR) têm como referência a linha entre as gotas de lágrima. a) $\mathrm{H} 1$ e $\mathrm{H} 2$ correspondem à aferição do eixo horizontal da hemipelve superior. Inicialmente é definida uma linha ligando os pontos mais superiores dos tetos acetabulares (linha supra-acetabular). $\mathrm{H} 1$ localiza-se $2 \mathrm{~cm}$ acima desta; $\mathrm{H} 2$ localiza-se $7 \mathrm{~cm}$ acima desta. b) V1 corresponde à aferição do eixo vertical da hemipelve superior. É a medida do ponto mais superior do osso ilíaco até a linha supra-acetabular. c) HR corresponde à aferição do eixo horizontal da hemipelve inferior, tendo como referência um ponto médio da articulação da sínfise púbica. d) VR corresponde à aferição do eixo vertical da hemipelve inferior, tendo como referência um ponto médio da aferição HR. 
As mensurações do eixo das hemipelves (H1, H2, V1, HR e VR) foram consideradas assimétricas quando, na comparação de uma hemipelve em relação ao lado contralateral, evidenciou-se uma diferença $>5 \mathrm{~mm}$.

Os pacientes selecionados foram separados em dois grupos: controle e grupo 1 .

No grupo controle foram incluídos pacientes cujos quadris apresentavam ângulo $C E$ com diferença $<5^{\circ}$, sem sinal do cruzamento das linhas acetabulares. No grupo 1 , foram incluídos pacientes com assimetria do ângulo $C E \geq 5^{\circ}$, na comparação de um quadril em relação ao lado contralateral, sem sinal do cruzamento das linhas acetabulares.

O objetivo do presente estudo é avaliar a correlação entre a variação do ângulo $\mathrm{CE}$ e a deformidade dos ossos pélvicos (assimetria de H1, H2, V1, HR e/ou VR), comparando-se ambos os grupos.

A hipótese é de que a presença de variação do ângulo $C E$ correlaciona-se com assimetria da hemipelve superior (sugerindo alteração rotacional da hemipelve superior).

Para avaliar a homogeneidade entre os grupos, foi aplicado o teste de qui-quadrado para idade, gênero, ângulo CE e IA. O teste exato de Fisher foi empregado para análise das variáveis qualitativas intergrupos (H1, H2, V1, HR e VR). No presente estudo, as diferenças foram consideradas estatisticamente significantes quando $p<0,05$. Foi utilizado o software PASW Statistics for Windows, Version 18.0 (SPSS Inc. Chicago, IL, EUA) na análise estatística. Um total de 50 radiografias foi selecionado randomicamente para aferição do coeficiente к. Dois autores, Roos B. D. e Lima E. M. U., avaliaram as radiografias em tempos distintos com concordância interobservador de 0,72 .

\section{Resultados}

O total de pacientes avaliados no período foi de 228 (456 quadris). De acordo com os critérios estabelecidos, foram incluídos no presente estudo 93 pacientes, sendo 49 do sexo masculino e 44 do sexo feminino. Os pacientes excluídos estão representados na - Tabela 1. A idade média foi de 39,9 anos (variando de 20 a 60 anos, desvio padrão [DP] $=10,52$ ),

Tabela 1 Descrição dos pacientes excluídos no estudo

\begin{tabular}{|l|l|}
\hline Critérios de exclusão & Excluídos \\
\hline Cirurgia prévia de quadril ou coluna & 6 \\
\hline Artrose $\geq$ Tönnis 2 & 12 \\
\hline DDQ severa & 8 \\
\hline Sobrecobertura acetabular global & 14 \\
\hline Sinal do cruzamento das linhas acetabulares & 60 \\
\hline Assimetria de ÂCE 1 ou $2^{\circ}$ & 11 \\
\hline $\begin{array}{l}\text { Assimetria de ÂCE }+ \text { Retroversão } \\
\text { acetabular unilateral }\end{array}$ & 9 \\
\hline EPF ou Legg-Perthes-Calveé & 3 \\
\hline Radiografia sem qualidade adequada & 12 \\
\hline Total & 135 \\
\hline
\end{tabular}

Abreviações: ÂCE, ângulo CE; DDQ, displasia do desenvolvimento do quadril; EPF, epifisiolise proximal do fêmur. e o ângulo CE médio do quadril direito foi de $31,5^{\circ}$ (variando de $20^{\circ}$ a $40^{\circ}, \mathrm{DP}=5,30$ ) e do esquerdo foi de $32,3^{\circ}$ (variando de $20^{\circ}$ a $40^{\circ}, \mathrm{DP}=5,11$ ). 0 IA médio foi de 5,14 (variando de 0 a $10, \mathrm{DP}=2,97$ ) no quadril direito e 5,17 (variando de 0 a 10 , $\mathrm{DP}=3,09)$ no quadril esquerdo.

Um total de 38 pacientes foi incluído no grupo controle, com média das mensurações dos eixos H1, H2, V1, HR e VR representadas na - Tabela 2. Com relação à $\mathrm{H} 1$, foi constatada aferição assimétrica em 4 casos (10,5\%), à H2 em 5 (13,1\%), à V1 em 7 (18,4\%), à HR em 5 (13,1\%), e à VR em 1 caso (2,63\%).

No grupo 1, foram incluídos 55 pacientes. A média das mensurações dos eixos H1, H2, V1, HR e VR estão representadas na - Tabela 3. Com relação à $H 1$, foi constatada aferição assimétrica em 24 casos (43,6\%), à H2 em 50 (90,9\%), à V1 em 28 (50,9\%), à HR em 16 (29,09\%), e à VR em 8 casos (14,5\%) (-Figura 2).

Os grupos foram considerados homogêneos, por não apresentarem diferença com relação a gênero, idade, ângulo CE e IA $(p=0,086)$. Na comparação entre o grupo controle e o grupo 1 (-Tabela 4), observou-se diferença estatisticamente significativa para a assimetria das mensurações $\mathrm{H} 1$ $(p<0,001), \mathrm{H} 2(p<0,001)$ e V1 $(p=0,005)$. Para as medidas HR e VR, não observou-se diferença estatisticamente significativa $(p=0,082 ; p=0,077)$.

\section{Discussão}

Existe evidência crescente na literatura da associação de alterações morfológicas ósseas na região do quadril e o desenvolvimento de sintomas, além da possibilidade de evolução para degeneração condral articular. Estas alterações podem estar relacionadas ao fêmur, ao acetábulo ou a ambos. ${ }^{1}$ No lado acetabular, como alterações morfológicas frequentes pode-se encontrar a sobrecobertura (IFA) e a deficiência de cobertura (DDQ). ${ }^{2,3}$

Alguns autores têm proposto que os distúrbios do desenvolvimento do quadril não afetam somente o fêmur proximal e o acetábulo, como é evidenciado em patologias como IFA e DDQ mas sim em toda a pelve. ${ }^{1,10}$ Porém, não há consenso sobre quais deformidades ósseas pélvicas correlacionam-se com alterações morfológicas acetabulares. Os presentes autores acreditam que o melhor entendimento destes dismorfismos pode levar à maior facilidade no diagnóstico das patologias articulares do quadril.

Fujii et al, ${ }^{13}$ realizaram um trabalho avaliando o alinhamento rotacional no plano axial do osso ilíaco em tomografias computadorizadas (TCs) de pacientes portadores de DDQ e questionaram: se a deformidade rotacional estava presente na DDQ; se os ângulos de rotação correlacionavamse com a versão e inclinação acetabulares; e se os ângulos de rotação correlacionavam-se com a região de deficiência do acetábulo. Os resultados evidenciaram que a rotação interna (RI) do terço superior da hemipelve (osso ilíaco cranialmente à espinha iliaca ântero-inferior [EIAI]) correlaciona-se com 0 diagnóstico de DDQ. ${ }^{12}$ A RI do osso ilíaco em pacientes portadores de DDQ também foi observada por autores como Kumeta et al $^{13}$ e Suzuki, ${ }^{14}$ e acredita-se que com esta deformidade 0 acetábulo tende a rodar 
Deformidade dos ossos pélvicos Roos et al. 243

Tabela 2 Descrição do valor das aferições H1, H2, V1, HR e VR no grupo controle

\begin{tabular}{|c|c|c|c|c|c|c|c|c|c|c|c|}
\hline Grupos & & H1 D & $\mathrm{H} 1 \mathrm{E}$ & H2 D & $\mathrm{H} 2 \mathrm{E}$ & V1 D & V1 E & HR D & HR E & VR D & VR E \\
\hline \multirow[t]{40}{*}{ Controle } & 1 & 71 & 71 & 94 & 93 & 101 & 102 & 67 & 67 & 67 & 68 \\
\hline & 2 & 57 & 65 & 91 & 92 & 92 & 91 & 96 & 92 & 55 & 51 \\
\hline & 3 & 55 & 58 & 85 & 87 & 103 & 100 & 90 & 88 & 80 & 79 \\
\hline & 4 & 51 & 52 & 86 & 89 & 95 & 98 & 93 & 91 & 58 & 57 \\
\hline & 5 & 43 & 43 & 90 & 80 & 115 & 115 & 80 & 88 & 55 & 55 \\
\hline & 6 & 49 & 49 & 97 & 98 & 112 & 112 & 90 & 90 & 57 & 57 \\
\hline & 7 & 66 & 67 & 88 & 88 & 110 & 110 & 76 & 76 & 68 & 69 \\
\hline & 8 & 55 & 61 & 92 & 100 & 126 & 124 & 91 & 100 & 66 & 68 \\
\hline & 9 & 50 & 50 & 111 & 113 & 134 & 134 & 83 & 84 & 60 & 60 \\
\hline & 10 & 73 & 75 & 108 & 106 & 133 & 131 & 91 & 91 & 65 & 67 \\
\hline & 11 & 88 & 88 & 106 & 106 & 131 & 139 & 81 & 90 & 77 & 77 \\
\hline & 12 & 64 & 62 & 100 & 99 & 131 & 133 & 85 & 85 & 53 & 53 \\
\hline & 13 & 72 & 72 & 102 & 100 & 126 & 121 & 76 & 78 & 88 & 90 \\
\hline & 13 & 58 & 60 & 105 & 105 & 137 & 137 & 90 & 88 & 51 & 53 \\
\hline & 15 & 57 & 60 & 110 & 115 & 105 & 107 & 87 & 85 & 75 & 73 \\
\hline & 16 & 55 & 57 & 85 & 87 & 133 & 134 & 85 & 83 & 52 & 50 \\
\hline & 17 & 78 & 78 & 108 & 109 & 132 & 127 & 91 & 90 & 69 & 67 \\
\hline & 18 & 62 & 60 & 126 & 124 & 137 & 143 & 88 & 87 & 60 & 61 \\
\hline & 19 & 72 & 70 & 100 & 100 & 142 & 142 & 80 & 82 & 80 & 80 \\
\hline & 20 & 57 & 55 & 105 & 105 & 151 & 141 & 96 & 97 & 59 & 59 \\
\hline & 21 & 60 & 60 & 103 & 102 & 112 & 112 & 84 & 82 & 77 & 76 \\
\hline & 22 & 58 & 59 & 94 & 92 & 110 & 110 & 94 & 86 & 67 & 66 \\
\hline & 23 & 56 & 56 & 98 & 97 & 97 & 98 & 89 & 89 & 77 & 77 \\
\hline & 24 & 51 & 53 & 102 & 101 & 110 & 110 & 98 & 98 & 78 & 78 \\
\hline & 25 & 50 & 50 & 98 & 98 & 111 & 112 & 89 & 91 & 57 & 56 \\
\hline & 26 & 56 & 57 & 88 & 88 & 110 & 110 & 76 & 76 & 69 & 69 \\
\hline & 27 & 65 & 68 & 86 & 88 & 104 & 101 & 89 & 88 & 81 & 80 \\
\hline & 28 & 50 & 52 & 87 & 90 & 95 & 97 & 93 & 93 & 58 & 56 \\
\hline & 29 & 50 & 50 & 112 & 113 & 134 & 134 & 83 & 83 & 60 & 61 \\
\hline & 30 & 64 & 65 & 108 & 108 & 132 & 131 & 90 & 89 & 66 & 67 \\
\hline & 31 & 64 & 63 & 99 & 98 & 132 & 133 & 85 & 86 & 54 & 53 \\
\hline & 32 & 58 & 60 & 105 & 105 & 137 & 137 & 82 & 84 & 53 & 54 \\
\hline & 33 & 55 & 57 & 85 & 86 & 130 & 131 & 85 & 85 & 52 & 51 \\
\hline & 34 & 82 & 82 & 100 & 100 & 140 & 138 & 81 & 82 & 77 & 77 \\
\hline & 35 & 56 & 55 & 103 & 102 & 112 & 112 & 82 & 80 & 76 & 76 \\
\hline & 36 & 55 & 54 & 99 & 100 & 97 & 96 & 90 & 90 & 77 & 77 \\
\hline & 37 & 63 & 64 & 102 & 101 & 107 & 109 & 95 & 95 & 75 & 75 \\
\hline & 38 & 80 & 80 & 100 & 99 & 140 & 142 & 80 & 80 & 82 & 82 \\
\hline & Média & 60.68 & 61.53 & 99.05 & 119.89 & 119.84 & 86.34 & 86.55 & 66.61 & 66.45 & 98.89 \\
\hline & $\begin{array}{l}\text { Desvio } \\
\text { Padrão }\end{array}$ & 10.212 & 10.01 & 9.291 & 16.256 & 16.045 & 6.671 & 6.5 & 10.612 & 10.84 & 9.282 \\
\hline
\end{tabular}

Legenda: D, Quadril direito; E, Quadril esquerdo. 
244 Deformidade dos ossos pélvicos Roos et al.

Tabela 3 Descrição das aferições H1, H2, V1, HR e VR no grupo 1

\begin{tabular}{|c|c|c|c|c|c|c|c|c|c|c|c|}
\hline Grupos & & H1 D & $\mathrm{H} 1 \mathrm{E}$ & H2 D & $\mathrm{H} 2 \mathrm{E}$ & V1 D & V1 E & HR D & HR E & VR D & VR E \\
\hline \multirow{44}{*}{$\begin{array}{l}\text { Inclinação } \\
\text { Acetabular }\end{array}$} & 1 & 45 & 45 & 90 & 80 & 100 & 99 & 105 & 105 & 68 & 66 \\
\hline & 2 & 84 & 81 & 90 & 92 & 170 & 170 & 76 & 76 & 72 & 70 \\
\hline & 3 & 60 & 50 & 110 & 100 & 100 & 100 & 80 & 80 & 72 & 74 \\
\hline & 4 & 50 & 45 & 100 & 94 & 100 & 105 & 96 & 96 & 64 & 63 \\
\hline & 5 & 62 & 62 & 107 & 100 & 113 & 115 & 87 & 87 & 72 & 72 \\
\hline & 6 & 55 & 50 & 104 & 100 & 102 & 104 & 88 & 88 & 77 & 75 \\
\hline & 7 & 53 & 58 & 72 & 89 & 105 & 109 & 75 & 73 & 63 & 70 \\
\hline & 8 & 60 & 54 & 92 & 86 & 112 & 110 & 88 & 86 & 67 & 66 \\
\hline & 9 & 56 & 57 & 110 & 117 & 110 & 115 & 97 & 107 & 64 & 62 \\
\hline & 10 & 50 & 50 & 90 & 98 & 100 & 100 & 70 & 74 & 67 & 65 \\
\hline & 11 & 73 & 64 & 107 & 97 & 112 & 112 & 69 & 70 & 65 & 65 \\
\hline & 12 & 57 & 51 & 92 & 93 & 107 & 107 & 80 & 79 & 70 & 68 \\
\hline & 13 & 50 & 58 & 92 & 97 & 128 & 123 & 90 & 92 & 47 & 47 \\
\hline & 13 & 82 & 73 & 114 & 110 & 134 & 139 & 93 & 90 & 77 & 77 \\
\hline & 15 & 69 & 69 & 120 & 124 & 138 & 143 & 90 & 80 & 84 & 75 \\
\hline & 16 & 55 & 60 & 118 & 128 & 146 & 145 & 88 & 87 & 52 & 50 \\
\hline & 17 & 52 & 58 & 76 & 80 & 127 & 124 & 89 & 89 & 59 & 59 \\
\hline & 18 & 70 & 70 & 112 & 119 & 135 & 138 & 90 & 90 & 58 & 60 \\
\hline & 19 & 55 & 48 & 115 & 100 & 128 & 108 & 85 & 83 & 40 & 42 \\
\hline & 20 & 67 & 83 & 116 & 120 & 136 & 136 & 75 & 66 & 55 & 53 \\
\hline & 21 & 61 & 62 & 118 & 109 & 135 & 143 & 85 & 75 & 87 & 72 \\
\hline & 22 & 51 & 40 & 85 & 92 & 124 & 127 & 83 & 86 & 57 & 57 \\
\hline & 23 & 49 & 49 & 148 & 123 & 144 & 146 & 95 & 93 & 60 & 60 \\
\hline & 24 & 80 & 82 & 104 & 97 & 119 & 120 & 73 & 75 & 85 & 83 \\
\hline & 25 & 47 & 46 & 82 & 88 & 118 & 116 & 86 & 84 & 57 & 55 \\
\hline & 26 & 53 & 53 & 101 & 96 & 109 & 132 & 82 & 84 & 75 & 73 \\
\hline & 27 & 69 & 69 & 102 & 98 & 138 & 129 & 92 & 90 & 60 & 58 \\
\hline & 28 & 62 & 60 & 95 & 94 & 134 & 135 & 95 & 94 & 62 & 61 \\
\hline & 29 & 42 & 42 & 94 & 102 & 144 & 149 & 102 & 95 & 72 & 67 \\
\hline & 30 & 55 & 55 & 98 & 90 & 145 & 153 & 94 & 92 & 85 & 84 \\
\hline & 31 & 60 & 60 & 75 & 81 & 113 & 112 & 82 & 83 & 85 & 73 \\
\hline & 32 & 62 & 55 & 104 & 94 & 98 & 104 & 70 & 62 & 66 & 64 \\
\hline & 33 & 57 & 61 & 102 & 108 & 108 & 104 & 86 & 88 & 77 & 77 \\
\hline & 34 & 60 & 61 & 96 & 102 & 102 & 102 & 68 & 68 & 72 & 72 \\
\hline & 35 & 44 & 44 & 83 & 91 & 97 & 97 & 77 & 76 & 65 & 65 \\
\hline & 36 & 72 & 72 & 119 & 124 & 137 & 143 & 90 & 82 & 83 & 81 \\
\hline & 37 & 55 & 61 & 120 & 128 & 145 & 145 & 87 & 87 & 51 & 45 \\
\hline & 38 & 52 & 59 & 75 & 80 & 127 & 123 & 89 & 89 & 72 & 71 \\
\hline & 39 & 67 & 67 & 113 & 119 & 135 & 139 & 92 & 90 & 58 & 60 \\
\hline & 40 & 56 & 48 & 110 & 100 & 128 & 108 & 85 & 83 & 41 & 42 \\
\hline & 41 & 66 & 83 & 116 & 121 & 136 & 136 & 71 & 67 & 54 & 44 \\
\hline & 42 & 61 & 63 & 117 & 109 & 136 & 143 & 85 & 76 & 74 & 72 \\
\hline & 43 & 51 & 41 & 86 & 92 & 124 & 127 & 82 & 86 & 60 & 60 \\
\hline & 44 & 49 & 48 & 140 & 123 & 144 & 144 & 95 & 95 & 58 & 58 \\
\hline
\end{tabular}


Tabela 3 (Continued)

\begin{tabular}{|c|c|c|c|c|c|c|c|c|c|c|c|}
\hline Grupos & & H1 D & $\mathrm{H} 1 \mathrm{E}$ & H2 D & $\mathrm{H} 2 \mathrm{E}$ & V1 D & V1 E & HR D & HR E & VR D & VR E \\
\hline & 45 & 80 & 82 & 104 & 96 & 119 & 121 & 73 & 74 & 85 & 83 \\
\hline & 46 & 48 & 46 & 82 & 89 & 118 & 116 & 87 & 84 & 55 & 58 \\
\hline & 47 & 54 & 53 & 101 & 95 & 109 & 132 & 82 & 84 & 74 & 73 \\
\hline & 48 & 70 & 69 & 102 & 98 & 138 & 129 & 92 & 91 & 59 & 58 \\
\hline & 49 & 44 & 42 & 94 & 101 & 143 & 149 & 98 & 92 & 65 & 66 \\
\hline & 50 & 50 & 50 & 98 & 89 & 145 & 153 & 94 & 93 & 70 & 69 \\
\hline & 51 & 60 & 61 & 76 & 81 & 113 & 113 & 83 & 83 & 75 & 73 \\
\hline & 52 & 62 & 55 & 104 & 93 & 98 & 104 & 70 & 62 & 62 & 61 \\
\hline & 53 & 57 & 62 & 101 & 108 & 108 & 104 & 86 & 86 & 72 & 72 \\
\hline & 54 & 61 & 61 & 96 & 101 & 103 & 103 & 68 & 66 & 60 & 63 \\
\hline & 55 & 45 & 44 & 83 & 92 & 97 & 96 & 77 & 77 & 60 & 59 \\
\hline & Média & 58.49 & 58.04 & 100.51 & 122.44 & 123.62 & 84.85 & 83.45 & 66.8 & 64.29 & 100.93 \\
\hline & Desvio Padrão & 10.011 & 11.41 & 13.179 & 17.359 & 18.27 & 9.093 & 9.867 & 11.453 & 10.544 & 15.608 \\
\hline
\end{tabular}

Legenda: D, Quadril direito; E, Quadril esquerdo.

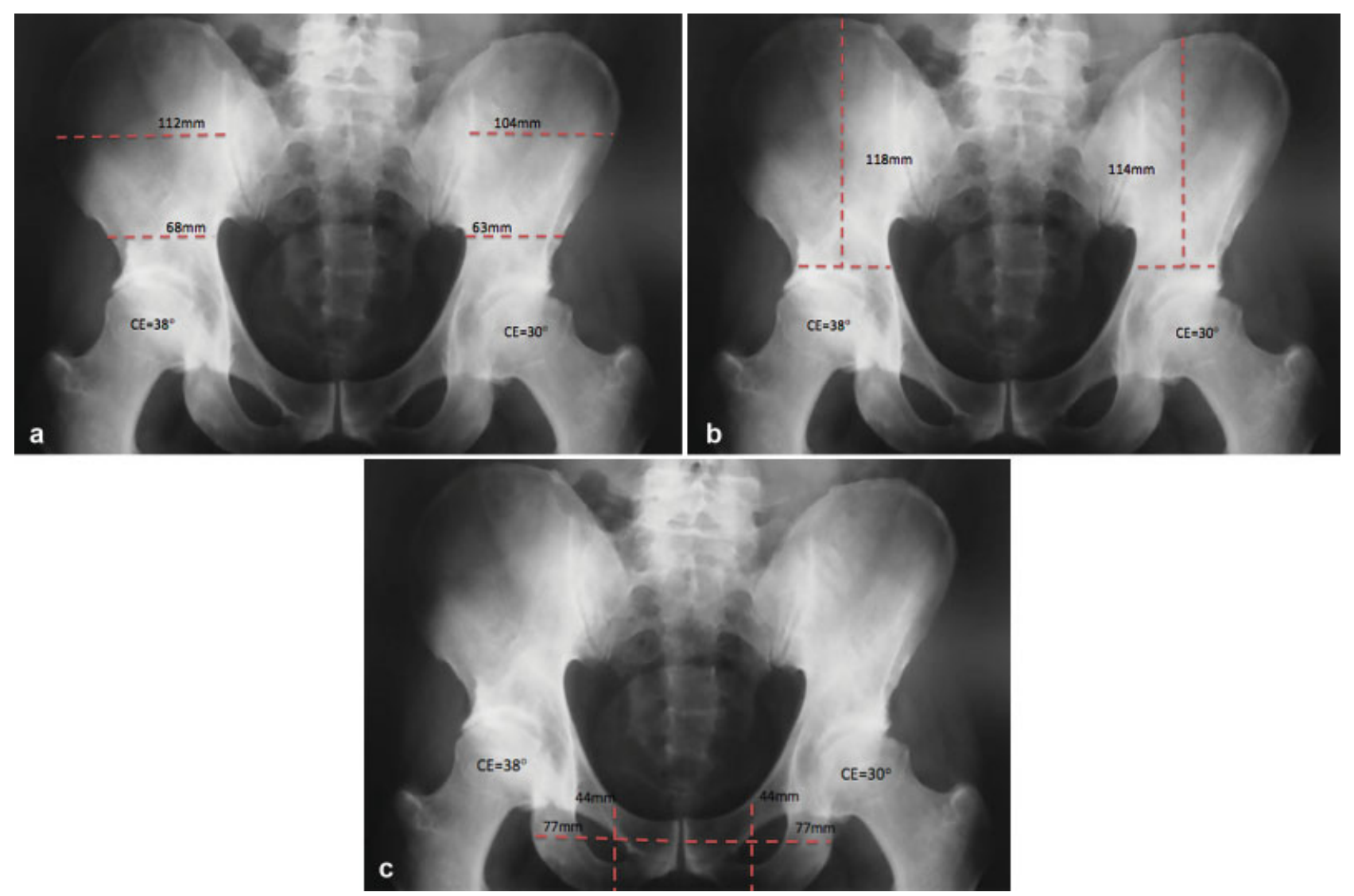

Fig. 2 Exemplificação de caso do grupo 1. a) Evidencia-se assimetria das aferições do eixo horizontal da hemipelve superior (H1 e H2). b) Evidencia-se assimetria da aferição do eixo vertical da hemipelve superior (V1). c) Evidencia-se simetria das aferições dos eixos horizontal e vertical da hemipelve inferior (HR e VR).

anterossuperiormente, resultando em diminuição da cobertura anterossuperior e aumento da cobertura posterior.

Também, Fujii et al $^{12}$ correlacionaram a rotação externa do terço inferior da hemipelve (entre o osso ilíaco e o ramo ísquiopúbico) com retroversão acetabular em pacientes portadores de DDQ. Este achado é corroborado por Kalberer et al, ${ }^{15}$ que observaram a proeminência da espinha isquiática em pacientes com retroversão do acetábulo. 
Tabela 4 Descrição dos resultados da comparação das aferições simétricas e assimétricas de H1, H2, V1, HR e VR, nos grupo controle versus grupo 1

\begin{tabular}{|c|c|c|c|c|c|c|c|c|c|c|c|}
\hline & \multicolumn{2}{|l|}{$\mathrm{H} 1$} & \multicolumn{2}{|l|}{$\mathrm{H} 2$} & \multicolumn{2}{|l|}{ V1 } & \multicolumn{2}{|l|}{ VR } & \multicolumn{2}{|l|}{$\mathrm{HR}$} & \multirow[b]{2}{*}{ Total } \\
\hline Assimetria & Sim & Não & Sim & Não & Sim & Não & Sim & Não & Sim & Não & \\
\hline Inclinação & 24 & 31 & 50 & 5 & 28 & 27 & 0 & 0 & 16 & 39 & 55 \\
\hline Acetabular & $85.70 \%$ & $47.70 \%$ & $90.90 \%$ & $13.20 \%$ & $77.80 \%$ & $47.40 \%$ & $0.00 \%$ & $0.00 \%$ & $76.20 \%$ & $54.20 \%$ & $59.10 \%$ \\
\hline \multirow[t]{2}{*}{ Controle } & 4 & 34 & 5 & 33 & 8 & 30 & 0 & 0 & 5 & 33 & 38 \\
\hline & $14.30 \%$ & $52.30 \%$ & $9.10 \%$ & $86.80 \%$ & $12.20 \%$ & $52.60 \%$ & $0.00 \%$ & $0.00 \%$ & $13.80 \%$ & $45.80 \%$ & $40.90 \%$ \\
\hline \multirow[t]{2}{*}{ Total } & 28 & 65 & 55 & 38 & 36 & 57 & 0 & 0 & 21 & 69 & 88 \\
\hline & $100 \%$ & $100 \%$ & $100 \%$ & $100 \%$ & $100 \%$ & $100 \%$ & $100 \%$ & $100 \%$ & $100 \%$ & $100 \%$ & $100 \%$ \\
\hline Significância & \multicolumn{2}{|c|}{$p=0,001$} & \multicolumn{2}{|c|}{$p<0,001$} & \multicolumn{2}{|c|}{$p=0,005$} & \multicolumn{2}{|c|}{$p=000$} & \multicolumn{2}{|l|}{$p=0,82$} & \\
\hline
\end{tabular}

Estas observações sugerem que alterações estruturais ósseas da pelve e anormalidades acetabulares não são achados isolados, e fazem parte de um continuum de alterações estruturais do desenvolvimento.

No presente trabalho, evidenciou-se correlação entre a variação do ângulo $\mathrm{CE}$ e assimetria da hemipelve superior. Assim como Fujii et al, ${ }^{12}$ podemos constatar que os dismorfismos da hemipelve superior, decorrentes de distúrbios do desenvolvimento ósseo, podem influenciar na morfologia acetabular. Porém, buscamos analisar estes achados em um grupo de pacientes sem DDQ severa (ÂCE $<20^{\circ}$ ).

Segundo nosso conhecimento, este é o primeiro estudo que busca estabelecer correlações entre dismorfismos pélvicos e variação do ângulo $\mathrm{CE}$, no percentil 90 para ângulo $\mathrm{CE}$ da população geral $\left(20-40^{\circ}\right) .^{16}$

\section{Conclusão}

No presente trabalho, evidenciou-se correlação entre a variação do ângulo CE e assimetria da hemipelve superior. Estes achados sugerem que os dismorfismos da hemipelve superior, decorrentes de distúrbios do desenvolvimento ósseo, podem influenciar na morfologia acetabular.

Conflito de Interesses

Os autores declaram não haver conflito de interesses.

\section{Referências}

1 Gosvig KK, Jacobsen S, Sonne-Holm S, Palm H, Troelsen A. Prevalence of malformations of the hip joint and their relationship to sex, groin pain, and risk of osteoarthritis: a population-based survey. J Bone Joint Surg Am 2010;92(05):1162-1169

2 Agricola R, Heijboer MP, Roze RH, et al. Pincer deformity does not lead to osteoarthritis of the hip whereas acetabular dysplasia does: acetabular coverage and development of osteoarthritis in a nationwide prospective cohort study (CHECK). Osteoarthritis Cartilage 2013;21(10):1514-1521
3 Reynolds D, Lucas J, Klaue K. Retroversion of the acetabulum. A cause of hip pain. J Bone Joint Surg Br 1999;81(02):281-288

4 Cooperman D. What is the evidence to support acetabular dysplasia as a cause of osteoarthritis? J Pediatr Orthop 2013;33(Suppl 1):S2-S7

5 Tannast M, Siebenrock KA, Anderson SE. Femoroacetabular impingement: radiographic diagnosis-what the radiologist should know. AJR Am J Roentgenol 2007;188(06):1540-1552

6 Dora C, Leunig M, Beck M, Simovitch R, Ganz R. Acetabular dome retroversion: radiological appearance, incidence and relevance. Hip Int 2006;16(03):215-222

7 Jamali AA, Mladenov K, Meyer DC, et al. Anteroposterior pelvic radiographs to assess acetabular retroversion: high validity of the “cross-over-sign". J Orthop Res 2007;25(06):758-765

8 Murphy RJ, Subhawong TK, Chhabra A, Carrino JA, Armand M, Hungerford M. A quantitative method to assess focal acetabular overcoverage resulting from pincer deformity using CT data. Clin Orthop Relat Res 2011;469(10):2846-2854

9 Jacobsen S, Sonne-Holm S. Hip dysplasia: a significant risk factor for the development of hip osteoarthritis. A cross-sectional survey. Rheumatology (Oxford) 2005;44(02):211-218

10 Albiñana J, Morcuende JA, Delgado E, Weinstein SL. Radiologic pelvic asymmetry in unilateral late-diagnosed developmental dysplasia of the hip. J Pediatr Orthop 1995;15(06):753-762

11 Clohisy JC, Carlisle JC, Beaulé PE, et al. A systematic approach to the plain radiographic evaluation of the young adult hip. J Bone Joint Surg Am 2008;90(Suppl 4):47-66

12 Kumeta H, Funayama K, Miyagi S, et al. Inward wing ilium of adult hip dysplasia a characteristic cross sectional pelvic anatomy visualized by CT. Rinsho Seikeigeka. 1986;21(01):67-75

13 Fujii M, Nakashima Y, Sato T, Akiyama M, Iwamoto Y. Pelvic deformity influences acetabular version and coverage in hip dysplasia. Clin Orthop Relat Res 2011;469(06):1735-1742

14 Suzuki S. Deformity of the pelvis in developmental dysplasia of the hip: three-dimensional evaluation by means of magnetic resonance image. J Pediatr Orthop 1995;15(06):812-816

15 Kalberer F, Sierra RJ, Madan SS, Ganz R, Leunig M. Ischial spine projection into the pelvis : a new sign for acetabular retroversion. Clin Orthop Relat Res 2008;466(03):677-683

16 Jacobsen S, Sonne-Holm S, Søballe K, Gebuhr P, Lund B. Hip dysplasia and osteoarthrosis: a survey of 4151 subjects from the Osteoarthrosis Substudy of the Copenhagen City Heart Study. Acta Orthop 2005;76(02):149-158 